\title{
Molecular dynamics study of the inhibitory effects of ChEMBL474807 on the enzymes GSK-3 $\beta$ and CDK-2
}

\author{
Przemyslaw Czeleń • Beata Szefler
}

Received: 24 September 2014 / Accepted: 15 February 2015 / Published online: 10 March 2015

(C) The Author(s) 2015. This article is published with open access at Springerlink.com

\begin{abstract}
Indirubin derivatives and analogs comprise a significant group of ATP-competitive inhibitors. The inhibitory effects of ChEMBL474807 (1-(4-amino-1,2,5-oxadiazol-3yl)-5-(piperidin-1-ylmethyl)- $N$ '-(pyridin-4-ylmethylene)- $1 H$ 1,2,3-triazole-4-carbohydrazide) on two enzymes, namely glycogen synthase kinase-3 $\beta$ (GSK-3 $\beta$ ) and cyclindependent kinase-2 (CDK-2), were analyzed. The close resemblance of the amino acid sequences of these two enzymes (with $25 \%$ identity and $41 \%$ similarity) explains why indirubin derivatives are inhibitors of both of the enzymes studied. The docking and molecular dynamics investigation performed here led to the identification of the interactions responsible for stabilizing the ligand ChEMBL474807 at the active sites of the enzymes considered. The structural and energetic data collected during our investigations clearly indicate that there are important differences in the behavior of the ligand at the two active sites investigated here.
\end{abstract}

Keywords ATPase $\cdot$ Competitive inhibition · Similarity · Molecular dynamics $\cdot$ CDK-2 - GSK-3 $\beta$

\section{Introduction}

Indirubin is a component of Indigo naturalis, namely the redcolored 3,2'-bisindole isomer. It is an active ingredient used in a traditional Chinese medicine for the treatment of chronic

This paper belongs to Topical Collection 6th conference on Modeling \& Design of Molecular Materials in Kudowa Zdrój (MDMM 2014)

P. Czeleń $(\bowtie) \cdot$ B. Szefler

Department of Physical Chemistry, Collegium Medicum, Nicolaus Copernicus University, Kurpinskiego 5, 85-950 Bydgoszcz, Poland e-mail: przemekcz@cm.umk.pl diseases. Small bioactive molecules that inhibit cellular replication machanisms are currently being exploited for novel therapeutics. Indirubin derivatives such as indirubin-3'-oxime have been investigated in this respect. Multiple effects of indirubin-3'-oxime on the activity of mitochondrial ATPase have been observed, indicating that this small molecule interferes with cell function [1]. Studies performed in vitro and on animals have shown that indirubin and its derivatives possess anti-inflammatory [2], antitumor [3, 4], antiangiogenic [5], and neuroprotective [6] effects. They also inhibit cyclindependent kinases in tumor cells [7, 8], enhance the cytotoxic effects of doxorubicin [9], and represent an efficient treatment for psoriasis [10]. In a study of patients with head and neck cancer, indirubins were found to reduce the mucosal damage caused by radiation therapy $[11,12]$. Indirubins inhibit DNA synthesis during cell proliferation in the late-G1 and G2/M phases by selectively inhibiting cyclin-dependent kinases (CDK) $[12,13]$ by competing at the ATPase-binding site [8]. Indirubin inhibits the assembly of microtubules involved in cell reproduction [14]. Its anti-inflammatory effects seem to originate from its inhibition of interferon gamma [2]. Indirubin is a constituent of Indigofera tinctoria; the synthetic form of indirubin was shown to have a similar effectiveness against CML to that of natural indirubin [15-17]. Indirubin also inhibits the growth of the human promyelocytic leukemia cell line HL-60 [18] and human HT-29 colorectal cancer cells [19], and it modulates the proliferation of keratinocytes in patients with psoriasis [20]. Mitochondrial dysfunction due to indirubin-3'-oxime may be an important mechanism for cell cycle arrest in human neuroblastoma cells [21].

During the investigations reported in the present paper, the inhibitory effects of ChEMBL474807 (1-(4-amino-1,2,5oxadiazol-3-yl)-5-(piperidin-1-ylmethyl)- $N$ '-(pyridin-4ylmethylene)-1 $H-1,2,3$-triazole-4-carbohydrazide) on two enzymes, namely glycogen synthase kinase-3 $\beta$ (GSK-3 $\beta$ ) and cyclin-dependent kinase-2 (CDK-2), were analyzed (see 
Fig. 1). The close resemblance of the amino acid sequences of these two enzymes (with $25 \%$ identity and $41 \%$ similarity) [22] proved that indirubin derivatives are inhibitors of both enzymes [23]. This situation necessitates a search for selective kinase-inhibiting compounds [24].

\section{Methods}

During the docking simulations, the crystal structures of CDK-2 (PDB ID: 1E9H) [25] and GSK-3 $\beta$ (PDB ID: 1Q41) were used [26], both of which were downloaded from the Brookhaven Protein Database (PDB). Docking was realized using a united-atom scoring function implemented in AutoDock Vina [27]. Before the docking stage, all of the water molecules were removed from the crystal structures of the considered enzymes, as were all of the nonpolar hydrogen atoms. All preparation steps were performed using the AutoDock Tools software package. After determining the grid box dimensions for each of the active sites considered, the docking procedure was repeated ten times. The conformations of the ligand ChEMBL474807 at the active sites of the

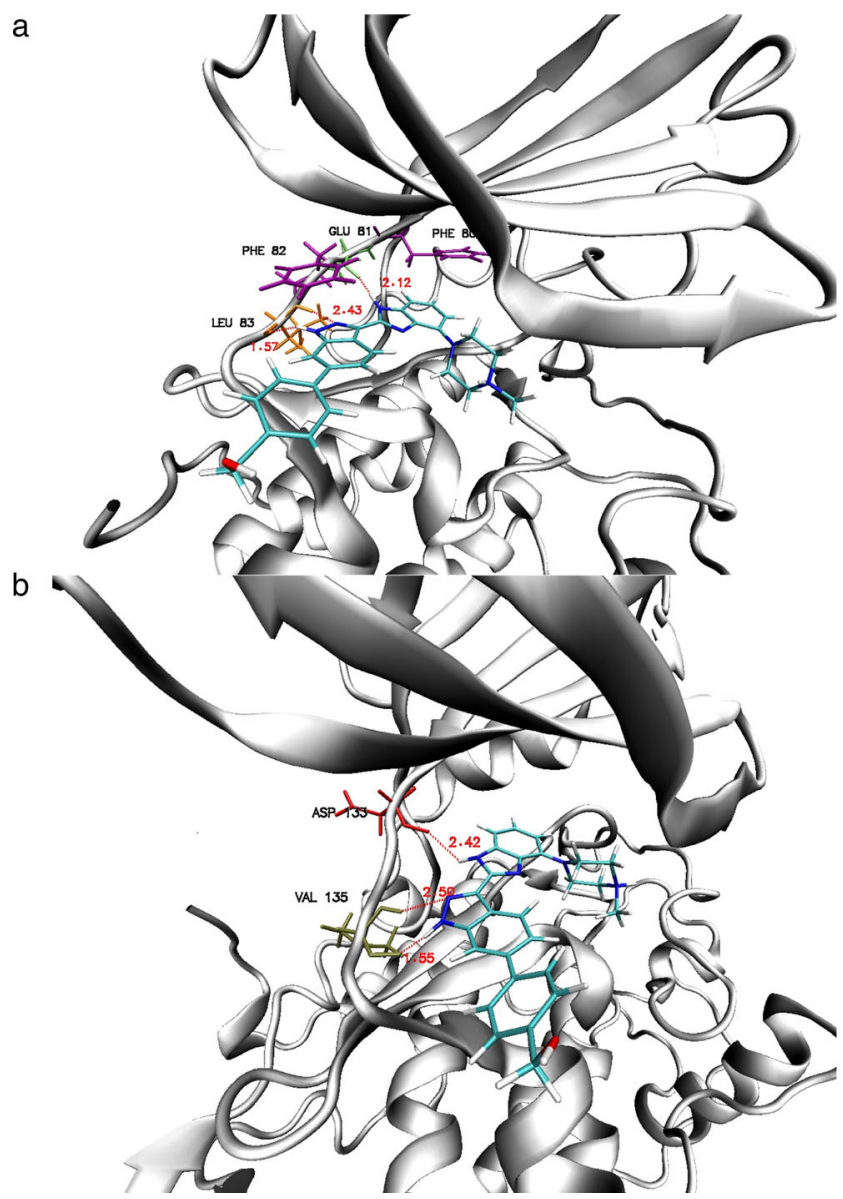

Fig. 1a-b The most important interactions observed between ChEMBL474807 and the active sites in the enzymes CDK-2 (a) and GSK-3 $\beta$ (b) enzymes (obtained during the docking stage) were almost identical, and reproduced the binding-site interactions observed experimentally [25, 26, 28, 29].

A molecular dynamics procedure was applied to the structure of the complex of the CDK-2 or GSK-3 $\beta$ protein subunit with the ligand ChEMBL474807, as obtained during the docking procedure. The structure of the ligand structure was characterized using Amber force-field parameters, and the atomic charges were calculated according to the MerzKollman scheme via the RESP procedure [30] at the HF/6$31 \mathrm{G}^{*}$ level. Each system was neutralized and immersed in a periodic TIP3P water box. The systems considered were heated to $300 \mathrm{~K}$ during the initial 100 ps of MD simulation, while the temperature was controlled by a Langevin thermostat [31]. Periodic boundary conditions and the SHAKE algorithm [32] were then applied for 110 ns of molecular dynamics simulation. The first $20 \mathrm{~ns}$ of this simulation time were used as an equilibration interval; the next $90 \mathrm{~ns}$ of the trajectory were used to analyze interactions between the subunits considered. The energies of the interactions between the ligand and the active site were characterized using the molecular mechanics/ Poisson-Boltzmann surface area (MMPBSA) method [33]. The AMBER 11 package [34] was used in all of the molecular dynamics simulations. Structural analysis, including calculations of the hydrogen bonds present and the root-mean-square deviations (RMSDs) of atomic positions, were performed using the VMD package [35]. All of the RMSD calculations for the ligand molecule and the amino acids at the active sites were performed relative to the initial structure of the complex obtained during the docking stage. Hydrogen atoms were excluded from those calculations.

\section{Results}

The proteins considered in this work, namely cyclindependent kinase 2 (CDK-2) and glycogen synthase kinase3 (GSK-3 $\beta$ ), exhibit high similarity in terms of the amino acid sequences within their active sites. The mechanism for inhibiting these enzymes has frequently been analyzed by computational methods. One group of compounds that exhibit high affinity for the ATP-binding pockets of the two kinases considered here includes indirubin and its analogs [22, 23, 25, 26, 28, 29]. Existing studies indicate a significant role of hydrogen bonds in stabilizing the interactions of these enzymes with ligands, with specific amino acids in each enzyme participating in the hydrogen bonds [28, 29]. For cyclindependent kinases, the most important roles in hydrogenbond creation are played by the amino acid residues GUA81 and LEU83; the former participates in one hydrogen bond while the latter is involved in two [28]. A very similar situation was observed for glycogen synthase kinases, where a network 


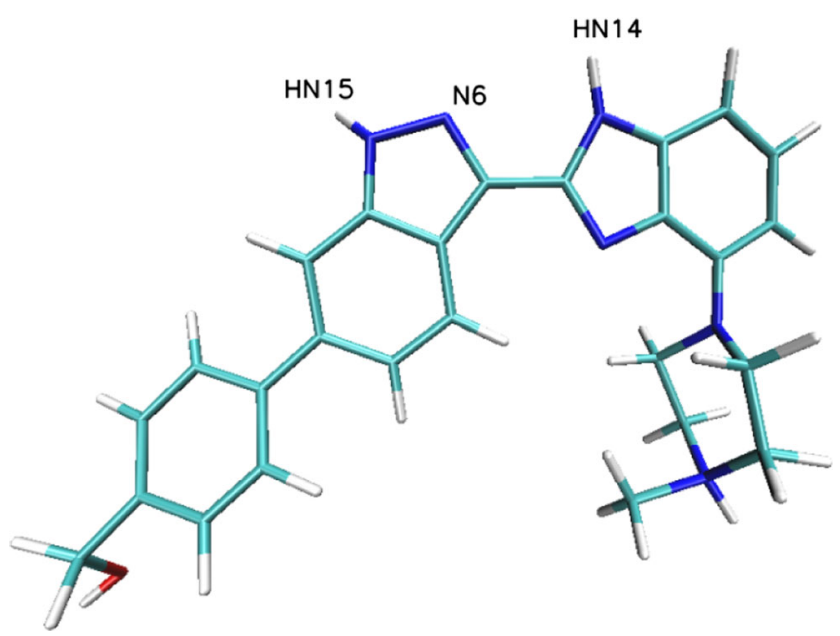

Fig. 2 Structure of the ligand molecule ChEMBL474807; the labeled atoms are involved in hydrogen bonds with amino acids from the active sites of CDK- 2 and GSK-3 $\beta$

of hydrogen bonds was created that involved ASP133 (one Hbond) and VAL135 (two H-bonds) [28, 29].

During the docking stage of our investigations, we used the molecule of the ligand ChEMBL474807 (Fig. 2). The nitrogen N6 and the hydrogens HN14 and HN15 were found to participate in hydrogen bonds that stabilize complexes of this ligand with kinases. After performing the docking procedure, complexes of both kinases with ChEMBL474807 were obtained. For both of the considered kinases, the ligand molecule was observed to be stabilized by three hydrogen bonds (see

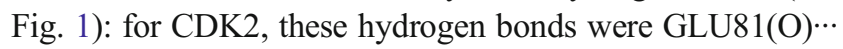

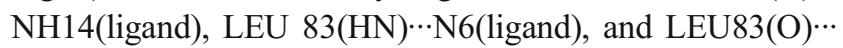
HN15(ligand); for GSK $3 \beta$, the hydrogen bonds were

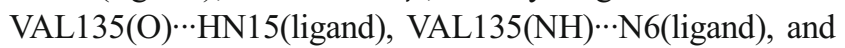
ASP133(O) $\cdots$ HN14(ligand). In both cases, the observed hydrogen bonds were consistent with existing findings for the interactions that occur in the ATP-binding pockets of the considered kinases. The binding affinities for both complexes were quite similar: $-9.1 \mathrm{kcal} / \mathrm{mol}$ for $\mathrm{CDK}-2$ and $-10.1 \mathrm{kcal} /$ mol for GSK- $3 \beta$. In both cases, the standard deviations for these values did not exceed $0.1 \mathrm{kcal} / \mathrm{mol}$.

The stabilities of the complexes obtained during the docking stage were evaluated by performing molecular dynamics simulations. These computations yielded a large group of conformations corresponding to the time evolution of

Table 1 Average RMSDs for the ligand and for the amino acids comprising the active site across the full molecular dynamics simulation

\begin{tabular}{lllll}
\hline & $\begin{array}{l}\text { Ligand when } \\
\text { complexed with } \\
\text { CDK-2 }\end{array}$ & $\begin{array}{l}\text { Active } \\
\text { site of } \\
\text { CDK-2 }\end{array}$ & $\begin{array}{l}\text { Ligand when } \\
\text { complexed with } \\
\text { GSK-3 } \beta\end{array}$ & $\begin{array}{l}\text { Active site } \\
\text { of GSK-3 } \beta\end{array}$ \\
\hline RMSD & 1.18 & 1.80 & 1.47 & 2.11 \\
SD & 0.18 & 0.21 & 0.19 & 0.25 \\
\hline
\end{tabular}

SD standard deviation
ChEMBL474807 at the active site of CDK- 2 or GSK- $3 \beta$. The stabilities of the structures formed during the trajectories in the simulations were evaluated by calculating their mean RMSD (root-mean-square deviation) values. The graphs presented in Fig. 3 show the time evolutions of the RMSDs for the ligand molecule and for all of the amino acids comprising the ATP-binding pockets in both kinases. The RMSD values
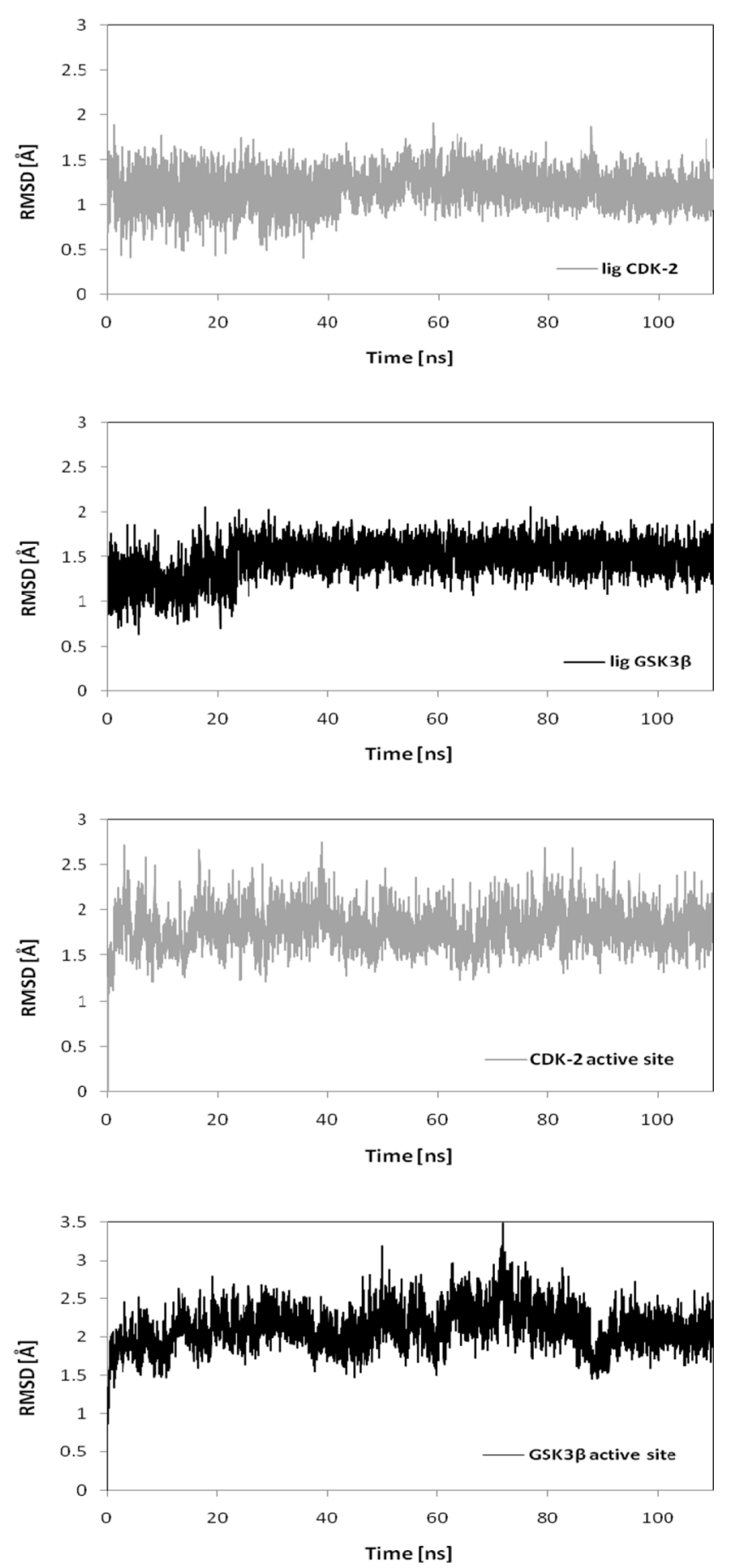

Fig. 3 Variations in the RMSD values for the ligand and for the amino acids of the active sites of CDK- 2 and GSK- $3 \beta$ over the course of the molecular dynamics simulation 
obtained led us to conclude that 20 ns of molecular dynamics simulation are sufficient to achieve equilibration. Slightly higher fluctuations in the RMSD value were seen for the GSK3B complex, an observation confirmed by the average values listed in Table 1. However, structural stabilization was seen for both complexes.

The final $90 \mathrm{~ns}$ of the trajectories were used for structural analysis. The structures of both ligand-protein complexes are consolidated by various types of forces, the most important of which are hydrogen bonds and hydrophobic interactions. The results of molecular dynamics simulations confirmed the conclusions drawn from the docking results. All three hydrogen bonds between ChEMBL474807 and amino acids (GLU81 and LEU83) in CDK-2 were present throughout the simulation (Fig. 1a), although the strengths of these interactions varied over time. The strength of a hydrogen bond can be gauged from the distance between the donor and acceptor. In the ATPbinding pocket, the most stable interaction was observed to be LEU83(O) $\cdots$ HN15(ligand). In over $90 \%$ of the conformations encountered during the simulation, the interaction between these atoms was a strong or moderately strong hydrogen bond (Table 2, Fig. 4). This amino acid (LEU83) also participates in the moderately strong interaction LEU83(HN) $\cdots \mathrm{N} 6$ (ligand), the length of which corresponded to a hydrogen bond in over $75 \%$ of the conformations collected during the simulation. The final interaction considered was GLU81(O) $\cdots$ NH14(ligand). This interaction corresponded to a strong hydrogen bond in some conformations, but to a moderately strong H-bond in most conformations (70 \%).

An important influence on the stability of each complex was the coexistence of all three of the hydrogen bonds reported above within the ATP-binding pocket, as illustrated in Fig. 5a, $\mathrm{b}$, and $\mathrm{c}$ for the active site of CDK2: all three plots show uniform concentric distributions, which confirms that all interactions responsible for complex stabilization have uniform distribution during whole simulation without any important fluctuations indicating on conformational flexibility of analyzed system. The hydrogen-bond length data suggest that most of the conformations collected during the molecular dynamics simulations are stabilized by at least one strong and two moderately strong bonds; only a few conformations include weak hydrogen bonds. These observations confirm the high stability of the complexes obtained during the docking stage.

The next most important interactions involved in the stabilization of the ligand at the active site of CDK-2 are those between the aromatic phenylalanine rings and ChEMBL474807. The data collected (Fig. 6) indicate that, throughout the whole simulation, the distances of these aromatic rings from ChEMBL474807 and the relative orientations of the rings with respect to the ligand permit the occurrence of stabilizing interactions. During most of the simulation (PHE80: $70 \%$; PHE82: $85 \%$ of the conformations), the distances between the two phenylalanine rings and the aromatic system of the ligand did not exceed $4.5 \AA$, leading to overlapping rings and stacking interactions in both cases.
Fig. 4a-b Distribution of the lengths of hydrogen bonds between ChEMBL474807 and amino acids in the active site of CDK-2 (a) or GSK-3 $\beta$ (b) throughout the simulation time. The hydrogen-bond lengths have been binned into $0.25-\AA$ intervals (the length values shown represent the midpoints of the intervals)

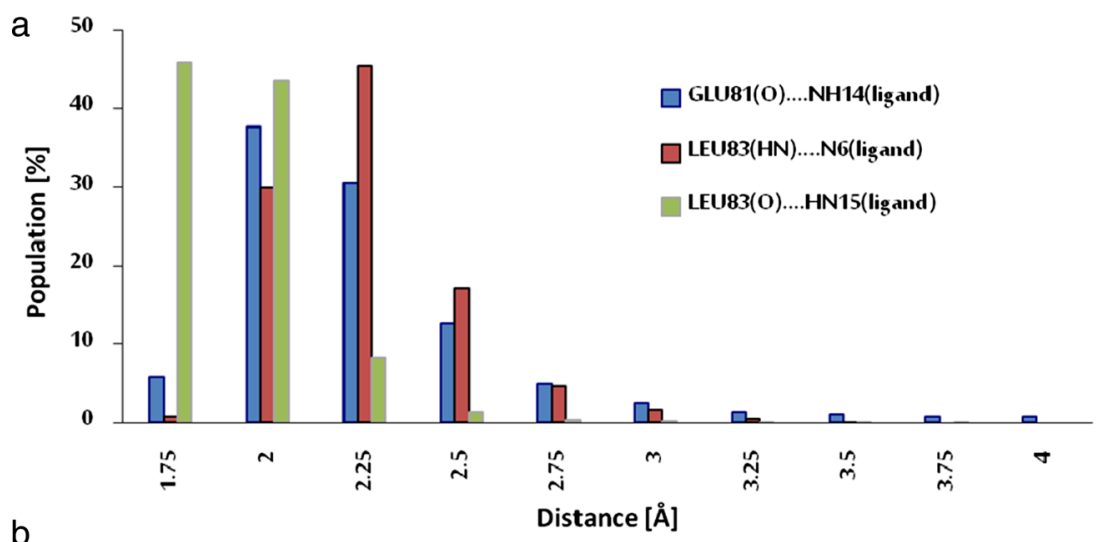

b

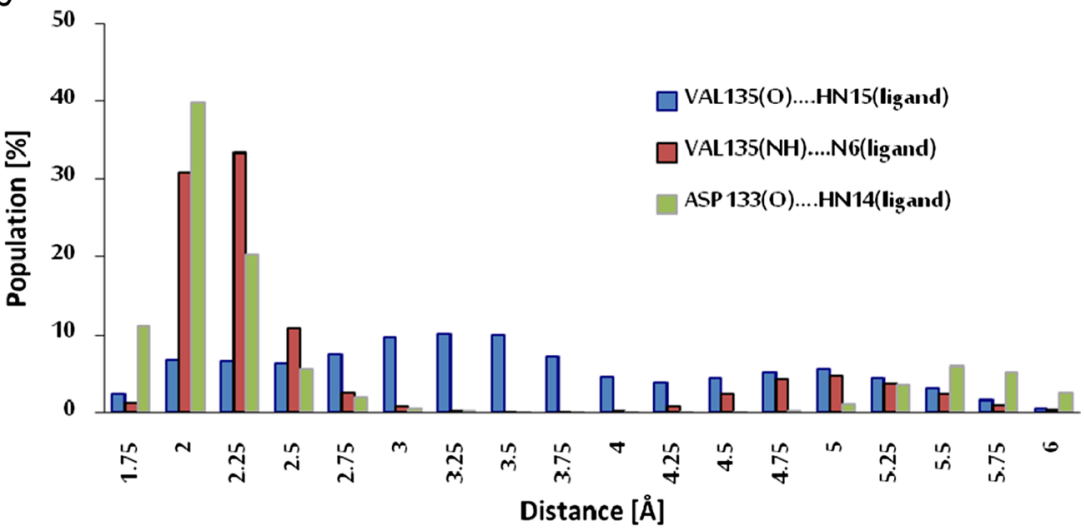


Fig. 5a-f Three hydrogen bonds occurred consistently in the complex ChEMBL474807CDK-2, according to the conformations collected during the molecular dynamics simulation of that complex, and three different $\mathrm{H}$-bonds occurred consistently during the corresponding simulation of the complex ChEMBL474807GSK-3 $\beta$. This figure presents plots of one hydrogen-bond length against another for the two complexes. H-bond lengths plotted for ChEMBL474807CDK-2: a

GLU81(O) $\cdots$ NH14(ligand)

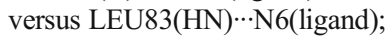
b LEU $83(\mathrm{HN}) \cdots \mathrm{N} 6$ (ligand) versus

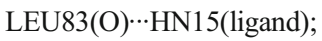

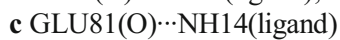
versus LEU83(O) ․HN15(ligand). $\mathrm{H}$-bond lengths plotted for ChEMBL474807-GSK-3 $\beta$ :

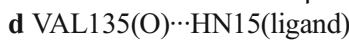
versus

VAL135(NH) $\cdots \mathrm{N} 6$ (ligand); e VAL135(O) $\cdots$ HN15(ligand) versus ASP133(O) $\cdots$ HN14(ligand);

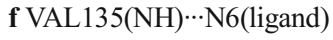
versus ASP133(O) $\cdots$ HN14(ligand)
CDK-2

a

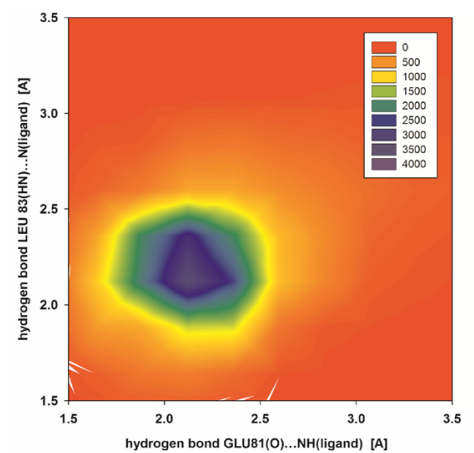

b

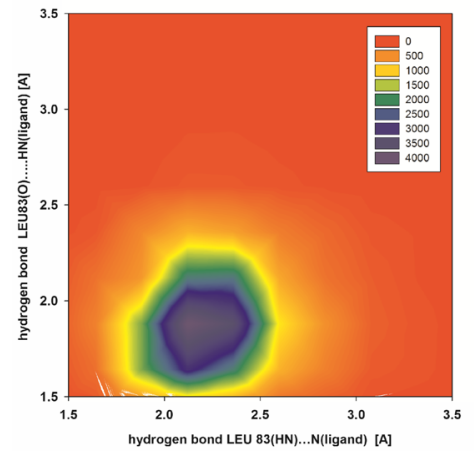

C

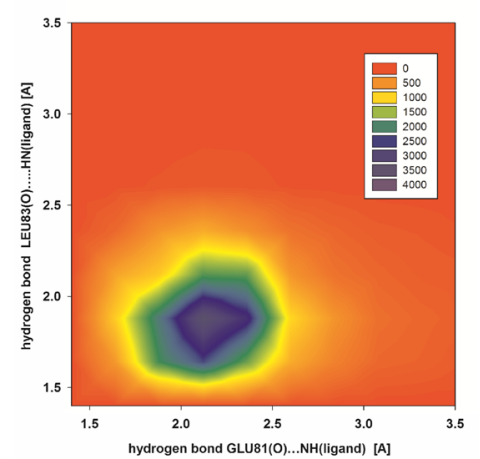

GSK-3 $\beta$

d

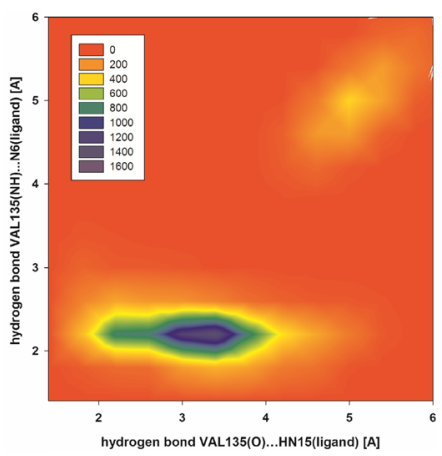

e

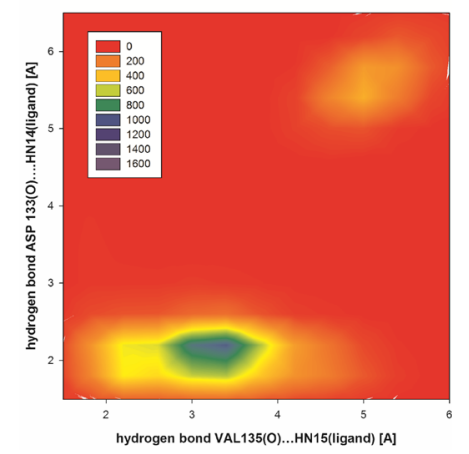

f

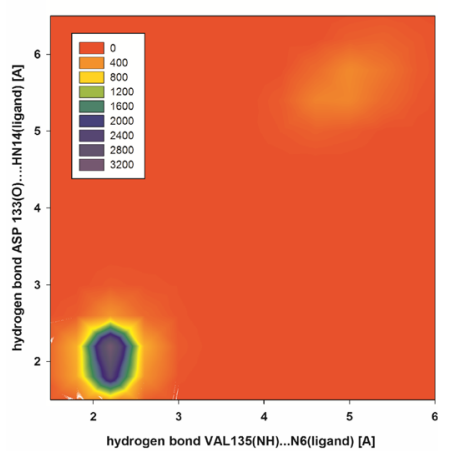

Interactions of ChEMBL474807 with the second active site, namely that of GSK-3 $\beta$, also involve three hydrogen bonds. VAL135 and ASP133 participate in these three $\mathrm{H}$ bonds (Fig. 1b). The most stable hydrogen bond is ASP133(O) $\cdots \mathrm{HN} 14($ ligand), which is present in more than $80 \%$ of the conformations produced during the simulation. While this bond was usually observed to be only moderately strong, there were a non-negligible group of conformations in which this interaction was strong (Table 2 and Fig. 4). The next most important H-bond between ChEMBL474807 and GSK-3 $\beta$ is VAL135(NH) $\cdots$ N6(ligand). This hydrogen bond was found to be moderately strong in $65 \%$ of the conformations; however, in about $20 \%$ of the conformations, the distance between the atoms involved in this bond exceeded $3 \AA$, which is too long for a hydrogen bond to be effective. The third and weakest H-bond between ChEMBL474807 and GSK-3 $\beta$ is VAL135(O) $\cdots \mathrm{HN} 15$ (ligand), observed in about
Fig. 6 Distribution of distances between ChEMBL474807 and two phenylalanine rings at the active site of CDK-2 
Table 2 Length distributions of the most common hydrogen bonds that occurred between ChEMBL474807 and selected amino acids from the active sites of CDK- 2 and GSK-3 $\beta$ in molecular dynamics simulations

\begin{tabular}{lll}
\hline Hydrogen bond considered & $\begin{array}{l}\text { Hydrogen- } \\
\text { bond length }(\AA)^{\mathrm{a}}\end{array}$ & $\begin{array}{l}\text { Population (i.e., } \\
\text { proportion of all } \\
\text { conformations, \% }\end{array}$
\end{tabular}

Between ChEMBL474807
and CDK-2

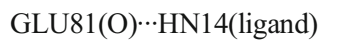

(1)

LEU83(HN) $\cdots \mathrm{N} 6$ (ligand)

\begin{tabular}{|c|c|c|}
\hline & 2.25 & 45.0 \\
\hline & 2.5 & 17.0 \\
\hline & 2.75 & 4.7 \\
\hline \multirow[t]{3}{*}{ LEU83(O) $\cdots$ HN15(ligand) } & 1.75 & 45.8 \\
\hline & 2 & 43.5 \\
\hline & 2.25 & 8.4 \\
\hline \multicolumn{3}{|l|}{$\begin{array}{l}\text { Between ChEMBL474807 } \\
\text { and GSK-3 } \beta\end{array}$} \\
\hline \multirow[t]{5}{*}{ VAL135(O) $\cdots$ HN15(ligand) } & 2 & 6.7 \\
\hline & 2.25 & 6.5 \\
\hline & 2.5 & 6.3 \\
\hline & 2.75 & 7.4 \\
\hline & 3 & 9.8 \\
\hline \multirow[t]{3}{*}{ 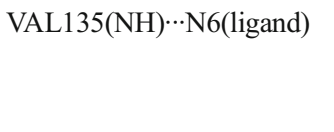 } & 2 & 31.0 \\
\hline & 2.25 & 33.0 \\
\hline & 2.5 & 11.0 \\
\hline \multirow[t]{4}{*}{ ASP133(O) $\cdots$ HN14(ligand) } & 1.75 & 11.1 \\
\hline & 2 & 40.0 \\
\hline & 2.25 & 20.3 \\
\hline & 2.5 & 5.7 \\
\hline
\end{tabular}

${ }^{a}$.In the table, hydrogen-bond lengths have been binned into $0.25-\AA$ length intervals; each length listed under 'Hydrogen-bond length' represents the midpoint of a length interval

$40 \%$ of the conformations collected during the molecular dynamics simulation. This H-bond was found to be either moderately strong or weak. The plots shown in Fig. $5 \mathrm{~d}$, e, and $\mathrm{f}$ confirm the previous observations made about the stabilizing interactions of ChEMBL474807 with GSK-3 $\beta$. The coexistence of the two hydrogen bonds ASP133(O) $\cdots$ HN14(ligand) and VAL135(NH) $\cdots$ N6(ligand) (Fig. 5f) was found to ensure a stable complex. The position of the center of the distribution and the shape of the distribution in each plot shed light on the binding interactions considered. The shapes of the distributions in Fig. 5d and e indicate that the VAL135(O) $\cdots$ HN15(ligand) bond fluctuates markedly in length during the simulation. In all of the charts (Fig. $5 \mathrm{~d}$, e, and $\mathrm{f}$ ), a minor fraction $(\sim 15 \%)$ of the conformations are characterized by interaction distances of 5-6 $\AA$. Interestingly,
Table 3 Binding free energies $(\Delta G, \mathrm{kcal} / \mathrm{mol})$ for the complex ChEMBL474807-CDK-2 and the complex ChEMBL474807-GSK$3 \beta$ during MD simulations. $\Delta H$ and $T \Delta S$ refer to the enthalpic and entropic contributions to the Gibbs free energy, respectively

\begin{tabular}{|c|c|c|c|c|c|c|}
\hline \multirow[t]{2}{*}{ Energetic parameter } & \multicolumn{2}{|l|}{ CDK-2 } & \multicolumn{2}{|c|}{ GSK-3 $\beta(1)^{\mathrm{a}}$} & \multicolumn{2}{|c|}{ GSK-3 $\beta(2)^{\mathrm{a}}$} \\
\hline & Value & SD & Value & SD & Value & SD \\
\hline$\Delta H$ & -28.29 & 4.13 & -26.01 & 3.92 & -17.53 & 3.15 \\
\hline$T \Delta S$ & -10.29 & 4.94 & -18.00 & 7.08 & -23.73 & 4.89 \\
\hline$\Delta G$ & -17.68 & 6.44 & -8.00 & 8.09 & 6.20 & 5.82 \\
\hline
\end{tabular}

For the complex involving GSK-3 $\beta$, two independent calculations were performed: first, the dominant conformations of the ligand relative to the active site were characterized [GSK-3 $\beta$ (1)]; second, the less common conformations were accounted for [GSK-3 $\beta$ (2)]

the hydrogen bonds differed in their length fluctuations. In the case of VAL135(O) $\cdots \mathrm{HN} 15$, unrestricted fluctuations covering the full range of bond lengths were observed, whereas stepwise changes in length were seen for the other two Hbonds (these stepped changes in bond length probably relate to changes in the conformation of the ligand ChEMBL474807 relative to the GSK-3 $\beta$ active site). These observations suggest the possibility of a presence of another energetically favorable conformation of ligand in the enzyme active site. Two hydrogen bonds, which formed between the ligand and oxygen atoms of two amino acids: VAL95(O) $\cdots \mathrm{HN} 14$ (ligand) and TYR94(OH) $\cdots$ N6(ligand), were observed. However analysis of the hydrogen-bond length, recorded for these conformations, suggests weak character of these interactions.

The energetics of both complexes during molecular dynamics simulation were analyzed. Gibbs free energy analysis was performed using the molecular mechanics/Poisson-Boltzmann surface area method. The enthalpy and entropy contributions to the Gibbs free energy were calculated for each complex.

Average energetic values for the interaction between ChEMBL474807 and CDK-2 and between ChEMBL474807 and GSK-3 $\beta$ are listed in Table 3. For the complex involving GSK-3 $\beta$, two independent calculations were done: first, the dominant conformations of the ligand relative to the active site were characterized; second, the less common conformations were accounted for. The stabilizing interactions observed within the complex of ChEMBL474807 with CDK-2 were confirmed because their presence corresponded to the lowest value of the Gibbs free energy. The $\Delta G$ values for the complex including GSK-3 $\beta$ indicated a low affinity of the ligand for the active site, especially in the second conformation analyzed.

\section{Conclusions}

Analysis of the properties of complexes formed by the ligand ChEMBL474807 with the kinases CDK-2 and GSK-3 $\beta$ 
revealed important differences between these complexes in their structural and energetic properties. For both complexes, conformations stabilized by hydrogen bonds (characteristic of indirubin and its analogs) were observed during the docking stage. However, the values obtained during molecular dynamics simulations indicated substantial differences between the behavior of the ligand ChEMBL474807 in the ATP-binding pocket of CDK-2 and its behavior in the ATP-binding pocket of GSK-3 $\beta$; these differences were mainly in the occurrence and strength of the hydrogen bonds between the ligand and each kinase. For the complex between ChEMBL474807 and the active site of CDK-2, the greatest contribution to the ligand-kinase binding derives from the heterocyclic part of the ligand molecule, namely the atoms HN15 and N6. On the other hand, for the complex between ChEMBL474807 and the active site of GSK-3 $\beta$, the heterocyclic part of the ligand molecule is much less involved in the binding process. The coexistence of all hydrogen bonds is a requirement for these complexes to remain stable. The disappearance or significant weakening of some of the H-bonds, as observed in the complex with GSK-3 $\beta$, may lead to structural distortions and conformational changes. The observed differences between the complexes, which are related to differences in the frequency of occurrence and strengths of particular hydrogen bonds as well as in binding affinities, indicate that there is a higher structural and energetic affinity of the ChEMBL474807 molecule for CDK-2 than for GSK-3 $\beta$.

In conclusion, the structural and energetic data presented here indicate a significant difference between the affinity of ChEMBL474807 for CDK-2 and the affinity of ChEMBL474807 for GSK-3 $\beta$.

Acknowledgments This project was supported by the PL-Grid Infrastructure.

Compliance with ethical standards Submitting an article "Molecular dynamics study of ChEMBL474807 inhibition properties against GSK3 and CDK2 enzymes" to Journal of Molecular Modeling does not have any sources of funding, as well as there are no potential conflicts of interest (financial or non-financial).

Open Access This article is distributed under the terms of the Creative Commons Attribution License which permits any use, distribution, and reproduction in any medium, provided the original author(s) and the source are credited.

\section{References}

1. Varela AT, Gomes AP, Simões AM, Teodoro JS, Duarte FV, Rolo AP, Palmeira CM (2008) Indirubin-3'-oxime impairs mitochondrial oxidative phosphorylation and prevents mitochondrial permeability transition induction. Toxicol Appl Pharmacol 233(2):179-185

2. Kunikata T, Tatefuji T, Aga H, Iwaki K, Ikeda M, Kurimoto M (2000) Indirubin inhibits inflammatory reactions in delayed-type hypersensitivity. Eur J Pharmacol 410:93-100
3. Kim SA, Kwon SM, Kim JA et al (2011) 5'-Nitro-indirubinoxime, an indirubin derivative, suppresses metastatic ability of human head and neck cancer cells through the inhibition of Integrin $\beta 1 / F A K / A k t$ signaling. Cancer Lett 306(2):197-204

4. Williams SP, Nowicki MO, Liu F et al (2011) Indirubins decrease glioma invasion by blocking migratory phenotypes in both the tumor and stromal endothelial cell compartments. Cancer Res 71(16):53745380

5. Shin EK, Kim JK (2012) Indirubin derivative E804 inhibits angiogenesis. BMC Cancer 12:164

6. Martin L, Magnaudeix A, Wilson CM, Yardin C, Terro F (2011) The new indirubin derivative inhibitors of glycogen synthase kinase-3, 6BIDECO and 6-BIMYEO, prevent tau phosphorylation and apoptosis induced by the inhibition of protein phosphatase-2A by okadaic acid in cultured neurons. J Neurosci Res 89(11):1802-1811

7. Marko D, Schatzle S, Friedel A, Genzlinger A, Zankl H, Meijer L et al (2001) Inhibition of cyclin-dependent kinase 1 (CDK1) by indirubin derivatives in human tumour cells. Br J Cancer 84:283-289

8. Hoessel R, Leclerc S, Endicott JA, Nobel ME, Lawrie A, Tunnah P et al (1999) Indirubin, the active constituent of a Chinese antileukaemia medicine, inhibits cyclin-dependent kinases. Nat Cell Biol 1:60-67

9. Shi R, Li W, Zhang X et al (2011) A novel indirubin derivative PHII7 potentiates Adriamycin cytotoxicity via inhibiting P-glycoprotein expression in human breast cancer MCF-7/ADR cells. Eur J Pharmacol 669(1-3):38-44

10. Lin YK, See LC, Huang YH et al (2012) Comparison of refined and crude indigo naturalis ointment in treating psoriasis: randomized, observer-blind, controlled, intrapatient trial. Arch Dermatol 148(3): 397-400

11. You WC, Hsieh CC, Huang JT (2009) Effect of extracts from indigowood root (Isatis indigotica Fort.) on immune responses in radiation-induced mucositis. J Altern Complement Med 15(7):771778

12. Xiao Z, Hao Y, Liu B, Qian L (2002) Indirubin and meisoindigo in the treatment of chronic myelogenous leukemia in China. Leuk Lymphoma 43:1763-1768

13. Kameswaran TR, Ramanibai R (2009) Indirubin-3-monooxime induced cell cycle arrest and apoptosis in Hep-2 human laryngeal carcinoma cells. Biomed Pharmacother 63(2):146-154

14. Steriti R (2002) Nutritional support for chronic myelogenous and other leukemias: a review of the scientific literature. Altern Med Rev 7:404-409

15. Han R (1994) Highlight on the studies of anticancer drugs derived from plants in China. Stem Cells 12:53-63

16. Zhang JT (2002) New drugs derived from medicinal plants. Therapie 57:137-150

17. Chen F, Li L, Ma D et al (2010) Imatinib achieved complete cytogenetic response in a CML patient received 32-year indirubin and its derivative treatment. Leuk Res 34(2):75-77

18. Cuong NM, Tai BH, Hoan DH et al (2010) Inhibitory effects of indirubin derivatives on the growth of HL-60 leukemia cells. Nat Prod Commun 5(1):103-106

19. Kim SH, Choi SJ, Kim YC, Kuh HJ (2009) Anti-tumor activity of noble indirubin derivatives in human solid tumor models in vitro. Arch Pharm Res 32(6):915-922

20. Lin YK, Leu YL, Yang SH et al (2009) Anti-psoriatic effects of Indigo naturalis on the proliferation and differentiation of keratinocytes with indirubin as the active component. J Dermatol Sci 54(3):168-174

21. Liao XM, Leung KN (2013) Indirubin-3'-oxime induces mitochondrial dysfunction and triggers growth inhibition and cell cycle arrest in human neuroblastomacells. Oncol Rep 29(1):371-379

22. terHaar E, Coll JT, Austen DA, Hsiao HM, Swenson L, Jain J (2001) Structure of GSK $3 \beta$ reveals a primed phosphorylation mechanism. Nat Struct Biol 8:593-596 
23. Dorronsoro I, Castro A, Martinez A (2002) Inhibitors of glycogen synthase kinase-3: future therapy for unmet needs? Expert Opin Ther Patents 12:1527-1536

24. Myrianthopoulos V, Magiatis P, Ferandin Y, Skaltsounis AL, Meijer L, Mikros E (2007) An integrated computational approach to the phenomenon of potent and selective inhibition of aurora kinases B and $\mathrm{C}$ by a series of 7-substituted indirubins. J Med Chem 50(17): 4027-4037

25. Davies TG, Tunnah P, Meijer L, Marko D, Eisenbrand G, Endicott JA, Noble ME (2001) Inhibitor binding to active and inactive CDK2: the crystal structure of CDK2-cyclin A/indirubin-5-sulphonate. Structure 9:389-397

26. Bertrand JA, Thieffine S, Vulpetti A, Cristiani C, Valsasina B, Knapp S, Kalisz HM, Flocco M (2003) Structural characterization of the GSK-3beta active site using selective and non-selective ATP-mimetic inhibitors. J Mol Biol 333:393-407

27. Trott O, Olson AJ (2010) AutoDock Vina: improving the speed and accuracy of docking with a new scoring function, efficient optimization and multithreading. J Comp Chem 31:455-461

28. Meijer L, Skaltsounis AL, Magiatis P, Polychronopoulos P, Knockaert M, Leost M, Xiaozhou XPR, Vonica CA, Brivanlou A, Dajani R, Crovace C, Tarricone C, Musacchio A, Roe SM, Pearl L, Greengard P (2003) GSK-3-selective inhibitors derived from Tyrian purple indirubins. Chem Biol 10:1255-1266

29. Bertrand JA, Thieffine S, Vulpetti A, Cristiani C, Valsasina B, Knapp S, Kalisz HM, Flocco M (2003) Structural characterization of the
GSK-3b active site using selective and non-selective ATP-mimetic inhibitors. J Mol Biol 333:393-407

30. Bayly CI, Cieplak P, Cornell WD, Kollman PA (1993) A wellbehaved electrostatic potential based method using charge restraints for deriving atomic charges: the RESP model. J Phys Chem 97: 10269-10280

31. Adelman SA, Doll JD (1976) Generalized Langevin equation approach for atom/solid-surface scattering: general formulation for classical scattering off harmonic solids. J Chem Phys 64(6):2375-2388

32. Ryckaert JP, Ciccotti G, Berendsen HJC (1977) Numerical integration of the Cartesian equations of motion of a system with constraints: molecular dynamics of $n$-alkanes. J Comput Phys 23(3):327-341

33. Miller BR, McGee TD, Swails JM, Homeyer N, Gohlke H, Roitberg AE (2012) MMPBSA.py: an efficient program for end-state free energy calculations. J Chem Theory Comput 8(9):3314-3321

34. Case DA, Darden TA, Cheatham TE III, Simmerling CL, Wang J, Duke RE, Luo R, Walker RC, Zhang W, Merz KM, Roberts B, Wang B, Hayik S, Roitberg A, Seabra G, Kolossváry I, Wong KF, Paesani F, Vanicek J, Liu J, Wu X, Brozell SR, Steinbrecher T, Gohlke H, Cai Q, Ye X, Wang J, Hsieh MJ, Cui G, Roe DR, Mathews DH, Seetin MG, Sagui C, Babin V, Luchko T, Gusarov S, Kovalenko A, Kollman PA (2010) AMBER 11. University of California, San Francisco

35. Humphrey W, Dalke A, Schulten K (1996) VMD: Visual Molecular Dynamics. J Mol Graphics 14:33-38 